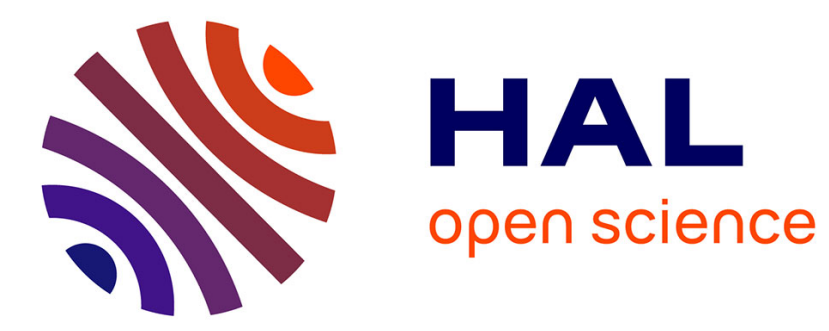

\title{
LIMBO Self-Test Method using binary input and dithering signals
}

Laurent Bourgois, Jérome Juillard

\section{To cite this version:}

Laurent Bourgois, Jérome Juillard. LIMBO Self-Test Method using binary input and dithering signals. Eurocon 2013 , Jul 2013, Zagreb, Croatia. pp.2111-2115, 10.1109/EUROCON.2013.6625272 . hal00862424

\section{HAL Id: hal-00862424 \\ https://hal-centralesupelec.archives-ouvertes.fr/hal-00862424}

Submitted on 16 Sep 2013

HAL is a multi-disciplinary open access archive for the deposit and dissemination of scientific research documents, whether they are published or not. The documents may come from teaching and research institutions in France or abroad, or from public or private research centers.
L'archive ouverte pluridisciplinaire HAL, est destinée au dépôt et à la diffusion de documents scientifiques de niveau recherche, publiés ou non, émanant des établissements d'enseignement et de recherche français ou étrangers, des laboratoires publics ou privés. 


\title{
LIMBO self-test method using binary input and dithering signals
}

\author{
Laurent Bourgois ${ }^{1}$, Jérôme Juillard ${ }^{2}$ \\ Department of Signal Processing and Electronic Systems, Supelec E3S \\ 3 rue Joliot-Curie, 91192 Gif-sur-Yvette Cedex, France \\ ${ }^{1}$ laurent.bourgois@supelec.fr \\ ${ }^{2}$ jerome.juillard@supelec.fr
}

\begin{abstract}
An online approach to system identification based on the least-mean squares (LMS) algorithm is presented in this paper. This recursive method is actually an extended version of the LMS-like identification method based on binary observations (LIMBO), whose practical requirement is a simple comparator (1-bit quantizer). This method can be applied in the case of finite impulse response (FIR) systems in the presence of noise and offset at the comparator input. Moreover, contrary to classical LIMBO approach, the unknown parameters are rigorously identified, and not up to a positive multiplicative constant. The idea consists in introducing a known dithering signal at the input of the quantizer, which acts as reference amplitude and allows us to identify the gain of the system. Some simulation results are given in order to compare the performances of this extended version of LIMBO with the usual one, in terms of convergence speed and estimation quality.
\end{abstract}

Index Terms-system identification, self-test, binary data processing, micro-systems

\section{INTRODUCTION}

Before their commercialization, micro-electro-mechanical systems (MEMS) must be tested to ensure that they will work properly under all operating conditions. Indeed, due to their very small characteristic dimension, these electronic devices are increasingly afflicted with variations in the fabrication process or environmental disturbances such as temperature, pressure and humidity fluctuations [1]. Typically, these tests are run after the fabrication process, but reveal to be very expensive and can even reach one-third of all production cost. To cut these costs, an alternative consists in implementing selftest features such as parameter estimation routines in each device. Obviously, these identification procedures must keep data storage to a minimum and have a low computational complexity to be implemented at a microscopic scale. Thus, parameter estimation methods based on binary observations are very appealing since they only involve the integration of a 1-bit analog-to-digital converter (ADC), which requires minimal design times and results in minimal silicon area and power consumption [2].

Several results have been established for system identification based on binary or roughly quantized observations in the last few years [3], [4], [5], [6], [7], [8], [9], [10]. A brief summary of these significant works can be found in [11], where an online LMS-like identification method based on binary observations has been introduced under the name of
LIMBO. This recursive parameter estimation procedure, which is naturally well-adapted to the context of micro-electronic devices, is derived from the offline WLS (weighted-least squares) approach developed in [1] and [12]. Its performances in terms of convergence speed and estimation quality are comparable to those obtained with the most of the previous works. Although LIMBO has already been put in practice for testing MEMS sensors [13], its convergence in the mean, with measurement noise at the input of the quantizer, has only been studied recently [14].

However, as things stand at the moment, LIMBO suffers from a drawback which is a direct consequence of the basic structure of its update procedure: it is only possible to estimate the parameters up to a positive multiplicative constant. This known issue has been reported in [1]. In this contribution, we propose to overcome this difficulty. For that, we add a known dithering signal at the comparator input, as it has been suggested in [5]. This dithering signal, which is very easy to generate in the context of micro-electronic applications by using a linear shift feedback register (LSFR) with the correct feedback relationship, acts as reference amplitude and allows us to identify the gain of the system. We then estimate the true parameter vector and not its normalized form. Moreover, we also take into account the estimation of the offset in the analysis by considering it as the mean of the measurement noise.

The structure of the article is the following. In section II, the system and its model are introduced. In section III, the extended LIMBO algorithm is derived. Then, the new proposed method is compared with the old one in terms of convergence speed and estimation quality. Some simulation results are graphically illustrated in section IV. Finally, concluding remarks and perspectives are given in section $\mathrm{V}$.

\section{FramewOrK AND Notations}

Let us consider the system illustrated in Fig. 1 below.

The input signal $u_{k}$ is filtered by a linear time-invariant discrete-time system $H$ to produce the system output $y_{k}$, where subscript $k$ indicates the discrete time. We assume that the transfer function has a finite impulse response of length $L$, i.e. the impulse response can be represented by a column vector $\boldsymbol{\theta}=\left(\theta_{l}\right)_{l=1}^{L}$. Consequently, the scalar value of the linear system output at time $k$ is given by $y_{k}=\boldsymbol{\theta}^{\mathrm{T}} \boldsymbol{\varphi}_{k, L}$ where $\boldsymbol{\varphi}_{k, L}=\left(u_{l}\right)_{l=k}^{k-L+1}$ 


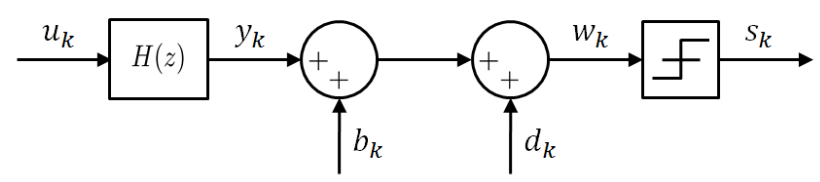

Fig. 1. Block diagram of the studied system.

is the regression column vector of dimension $L$. The system output is then measured via a 1-bit ADC so that only its sign $s_{k}=S\left(y_{k}+b_{k}+d_{k}\right)$ is available at time $k$. Here, $b_{k}$ corresponds to the additive measurement noise and $d_{k}$ refers to the known additive dithering signal. By keeping in mind that the cost must be minimized, the input and dithering signals are generated using a binary distribution. The function $S$ of a real number $x$ is characterized by:

$$
S(x)=\left\{\begin{aligned}
1 & \text { if } x \geq 0 \\
-1 & \text { otherwise }
\end{aligned}\right.
$$

We aim to develop a recursive estimation method to find a good estimate of both the impulse response $\boldsymbol{\theta}$ and the offset $o=\frac{1}{N} \sum_{k=1}^{N} b_{k}$, starting from $N$ observations of the binary output, knowing the input. Let $\hat{\boldsymbol{\theta}}_{k}$ and $\hat{o}_{k}$ be the estimated impulse response and offset, respectively. Thanks to the dithering signal, which acts as reference amplitude, we can also estimate the gain $G$ of the system. By denoting it $\hat{G}_{k}$, the estimated scalar value of the input of the quantizer at time $k$ is given by:

$$
\hat{w}_{k}=\hat{G}_{k}\left(\hat{\boldsymbol{\theta}}_{k}^{\mathrm{T}} \boldsymbol{\varphi}_{k, L}+\hat{o}_{k}+d_{k}\right)
$$

And the following relation can be established:

$$
\hat{w}_{k}=\hat{\tilde{\boldsymbol{\theta}}}_{k}^{\mathrm{T}} \boldsymbol{\varphi}_{k, L}+\hat{\tilde{\sigma}}_{k}+\hat{G}_{k} d_{k}
$$

where $\hat{\tilde{\boldsymbol{\theta}}}_{k}=\hat{G}_{k} \hat{\boldsymbol{\theta}}_{k}$ and $\hat{\tilde{o}}_{k}=\hat{G}_{k} \hat{o}_{k}$.

At this stage, it is more convenient to define the estimated augmented parameter vector $\hat{\tilde{\mathbf{h}}}_{k}$ as follows:

$$
\hat{\tilde{\mathbf{h}}}_{k}=\left[\begin{array}{c}
\hat{\tilde{\boldsymbol{\theta}}}_{k} \\
\hat{\tilde{o}}_{k} \\
\hat{G}_{k}
\end{array}\right]
$$

so that, by denoting:

$$
\boldsymbol{\psi}_{k, L}=\left[\begin{array}{c}
\varphi_{k, L} \\
1 \\
d_{k}
\end{array}\right]
$$

we can write:

$$
\hat{w}_{k}=\hat{\tilde{\mathbf{h}}}_{k}^{\mathrm{T}} \boldsymbol{\psi}_{k, L}
$$

Finally, let us also introduce the estimated sign of the system output $\hat{s}_{k}=S\left(\hat{w}_{k}\right)$.

\section{Proposed Approach}

By inspiring from the original version of LIMBO, we define the instantaneous error $\varepsilon_{k}=\left|s_{k}-\hat{s}_{k}\right| \hat{w}_{k}$. The general LMS algorithm is then performed to adjust the system parameters by minimizing this error. For that, the square of $\varepsilon_{k}$ is used as criterion. Indeed, $\varepsilon_{k}^{2}$ is clearly differentiable with respect to $\hat{w}_{k}$, which is also differentiable with respect to $\hat{\tilde{\mathbf{h}}}_{k}$. Consequently, this criterion is differentiable with respect to the system parameters, which yields:

$$
\begin{aligned}
\hat{\tilde{\mathbf{h}}}_{k+1} & =\hat{\tilde{\mathbf{h}}}_{k}-\frac{1}{2} \alpha_{k} \frac{\partial \varepsilon_{k}^{2}}{\partial \hat{\tilde{\mathbf{h}}}_{k}} \\
& =\hat{\tilde{\mathbf{h}}}_{k}-\frac{1}{2} \alpha_{k} \frac{\partial \varepsilon_{k}^{2}}{\partial \hat{w}_{k}} \frac{\partial \hat{w}_{k}}{\partial \hat{\tilde{\mathbf{h}}}_{k}} \\
& =\hat{\tilde{\mathbf{h}}}_{k}-4 \alpha_{k}\left[s_{k} \neq \hat{s}_{k}\right] \hat{w}_{k} \boldsymbol{\psi}_{k, L}
\end{aligned}
$$

where the notation $\left[s_{k} \neq \hat{s}_{k}\right]$ stands for a variable that is equal to unity if $s_{k} \neq \hat{s}_{k}$, and equal to zero otherwise.

In (7), $\alpha_{k}$ corresponds to the LMS step-size parameter that guarantees stability and controls the speed of convergence. In standard LIMBO, a procedure to determine an appropriate adaptive parameter has consisted in choosing $\alpha_{k}$ so that $\left\|\hat{\mathbf{h}}_{k}\right\|$ remains constant in time, where:

$$
\hat{\mathbf{h}}_{k}=\left[\begin{array}{c}
\hat{\boldsymbol{\theta}}_{k} \\
\hat{o}_{k}
\end{array}\right]
$$

In this manner, and by considering that $\left\|\hat{\mathbf{h}}_{1}\right\|=1$, the normalized parameter vector has been estimated without increasing the complexity of the algorithm. In our approach, the dithering signal allows us to estimate the gain of the system and, consequently, to estimate the true parameter vector instead of its normalized form. It is then not a priori imperative to follow the same path to determine $\alpha_{k}$. However, a slightly decreasing of $\left\|\hat{\tilde{\mathbf{h}}}_{k}\right\|$ is observable in the presence of measurement noise, which may lead to numerical instabilities. We then decide to choose $\alpha_{k}$ so that the norm of $\hat{\tilde{\mathbf{h}}}_{k}$ remains unchanged for all $k$, especially since this procedure is without consequence on the computational complexity. To this end, we use the following expression:

$$
\alpha_{k}=\frac{1}{2\left\|\psi_{k, L}\right\|^{2}}
$$

Finally, (9) is introduced into (7) to derive the parameter update equation:

$$
\hat{\tilde{\mathbf{h}}}_{k+1}=\hat{\tilde{\mathbf{h}}}_{k}-2\left[s_{k} \neq \hat{s}_{k}\right] \hat{w}_{k} \frac{\boldsymbol{\psi}_{k, L}}{\left\|\boldsymbol{\psi}_{k, L}\right\|^{2}}
$$

Once the estimation procedure has been performed, the true impulse response, as well as the true offset at the comparator input, are both straightforwardly obtained by dividing $\tilde{\boldsymbol{\theta}}_{\infty}$ and $\hat{\tilde{o}}_{\infty}$ by $\hat{G}_{\infty}$. 
Finally, although the extended LIMBO procedure requires the estimation of one additional parameters, it has still, by construction, a very low computational complexity and implementation cost. Besides, from a computational point of view, since $u_{k} \in\{-1,1\}$ and $d_{k} \in\{-1,1\}$, the update relation (10) can be simplified for $k>L$. Indeed, in this case, we have $\left\|\psi_{k, L}\right\|^{2}=L+2$.

\section{Results And Discussion}

In this section, the results obtained with our new version of LIMBO are compared in terms of convergence speed and estimation quality with those obtained by applying typical LIMBO procedure.

To this end, we consider an impulse response of length $L=100$ which takes the form of a damped sinusoid. The input signal is white and centered, with a Bernoulli distribution, and takes only the values 1 and -1 . The offset $o$ at the input of the comparator and the gain $G$ of the system are random parameters uniformly distributed on the interval $[0,1]$. The additive noise is also white and follows a Gaussian distribution with mean $o$ and variance $\sigma_{b}^{2}$. The dithering signal is generated following the same procedure as the input signal, and is consequently binary and entirely known. Lastly, the identification procedure detailed in section III is applied starting from $N=10^{5}$ observations of the binary output.

To efficiently measure the convergence speed and the estimation quality of the online estimation, the authors of LIMBO have introduced the sequence $\left(1-v_{k}\right)$, where $v_{k}$ is the cosine of the angle made by $\hat{\mathbf{h}}_{k}$ and $\mathbf{h}$. Without loss of generality, they have assumed that $\|\mathbf{h}\|=1$ in their simulations so that $v_{k}=\mathbf{h}^{\mathrm{T}} \hat{\mathbf{h}}_{k}$. Indeed, both vectors are then normalized, since $\left\|\hat{\mathbf{h}}_{k}\right\|=\left\|\hat{\mathbf{h}}_{1}\right\|=1$ for all $k$. Consequently, the following equivalence relation has been obtained:

$$
\lim _{k \rightarrow \infty}\left(1-v_{k}\right)=0 \Leftrightarrow \lim _{k \rightarrow \infty}\left(\hat{\mathbf{h}}_{k}\right)=\mathbf{h}
$$

In order to establish a comparable equivalence relation for our method, we follow the same protocol. For that, the vector $\tilde{\mathbf{h}}$ is beforehand normalized and our algorithm is initialized by setting $\hat{\tilde{\mathbf{h}}}_{1}=\left[\begin{array}{llll}1 & 0 & \cdots & 0\end{array}\right]^{\mathrm{T}}$ so that the norm of $\hat{\tilde{\mathbf{h}}}_{k}$ is set to 1 for all $k$. Thus, by denoting $\tilde{v}_{k}$ the cosine of the angle made by $\hat{\tilde{\mathbf{h}}}_{k}$ and $\tilde{\mathbf{h}}$, we have:

$$
\lim _{k \rightarrow \infty}\left(1-\tilde{v}_{k}\right)=0 \Leftrightarrow \lim _{k \rightarrow \infty}\left(\hat{\tilde{\mathbf{h}}}_{k}\right)=\tilde{\mathbf{h}}
$$

Note, however, that this particular choice is not imposed by our approach, in which $\hat{\tilde{\mathbf{h}}}_{1}$ may contains random initial values drawn from any given distribution function, but is only made in order to work with a similar sequence as the one used in LIMBO procedure.

By way of verification, we apply for each experiment a Monte Carlo simulation approach based on 5000 realizations of the input and dithering signals. The performances are then measured by computing the empirical mean of $\left(1-v_{k}\right)$ and $\left(1-\tilde{v}_{k}\right)$, respectively.
First, both methods are compared in a trivial case. For that, we suppose that system identification is achieved in the noisefree case, i.e. with $\sigma_{b}^{2}=0$. As it can be seen in Fig. 2, there is no significant difference in terms of estimation quality between LIMBO approach and its extended version.

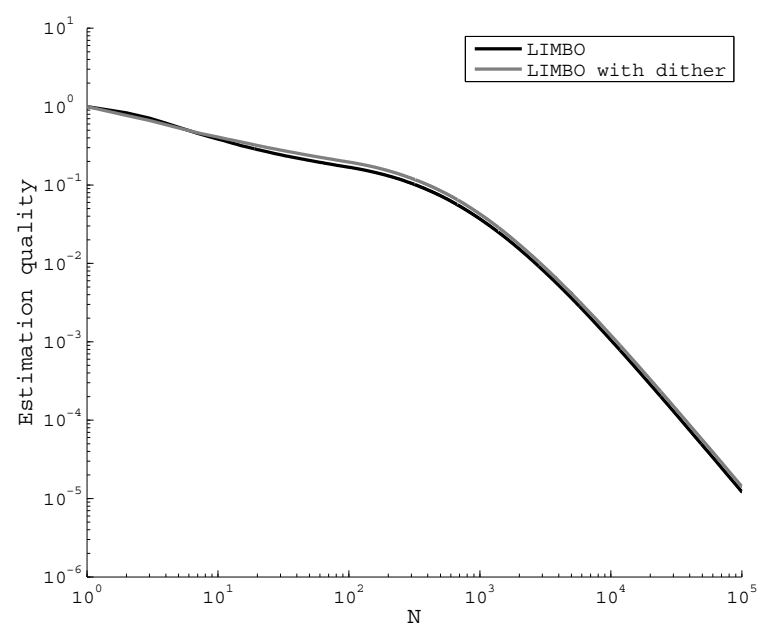

Fig. 2. Empirical mean of the quantities $\left(1-v_{k}\right)$ and $\left(1-\tilde{v}_{k}\right)$ in the noise-free case using a binary (Bernoulli) distributed input signal.

Indeed, these two methods have reached in mean an error level approximately equal to $10^{-5}$ at the end of the estimation procedure. Besides, the performances in terms of convergence speed are also similar, since the tangent of both curves is the same at each iteration point. Thus, the gain of the system, which acts as additional parameter to be conjointly estimated, does not degrade the performances of our approach.

At this point, we aim to evaluate the effect of perturbations. For that, system identification is achieved in the presence of measurement noise, whose variance value changes between $10^{-5}$ and $10^{-1}$. We still apply a Monte Carlo simulation approach and the signal-to-noise ratio (SNR) lies in average between about $50 \mathrm{~dB}$ and $5 \mathrm{~dB}$. The performances of the online estimation are shown in Fig. 3.

As expected, the presence of noise significantly degrades the performances in terms of estimation quality. Actually, the sequence stops decreasing after a certain error level which is approximately equal in mean to the variance of the noise. On the other hand, the performances in terms of convergence speed are similar whatever the noise level, since the tangent of each curve is approximatively the same at each iteration point until the corresponding error threshold has been reached. At last, there is once again no significant difference between LIMBO approach and its extended version.

In order to improve the performances in terms of estimation quality in presence of noise, a typical solution consists in using a relaxation procedure. For that, we define a constant relaxation parameter $0<\mu<1$ so that, at each update, we have: 


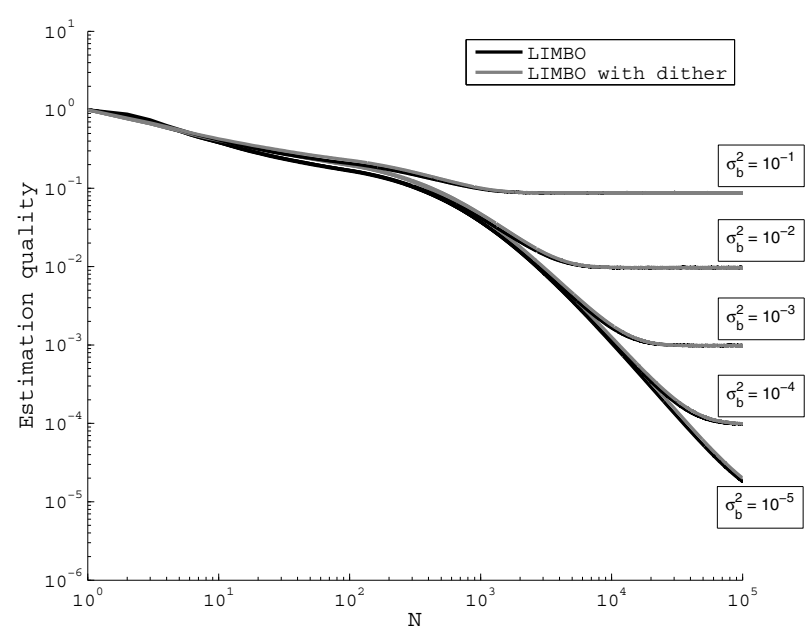

Fig. 3. Empirical mean of the quantities $\left(1-v_{k}\right)$ and $\left(1-\tilde{v}_{k}\right)$ considering a Gaussian distribution of the noise with several values of $\sigma_{b}^{2}$ and using a binary (Bernoulli) distributed input signal.

$$
\hat{\tilde{\mathbf{h}}}_{k+1}^{r l x}=\mu \hat{\tilde{\mathbf{h}}}_{k+1}+(1-\mu) \hat{\tilde{\mathbf{h}}}_{k}
$$

where $\hat{\tilde{\mathbf{h}}}_{k+1}^{r l x}$ is the relaxed solution at time $k+1$. Next, by introducing (10) into (13), we obtain the following update relation:

$$
\hat{\tilde{\mathbf{h}}}_{k+1}^{r l x}=\hat{\tilde{\mathbf{h}}}_{k}-2 \mu\left[s_{k} \neq \hat{s}_{k}\right] \hat{w}_{k} \frac{\boldsymbol{\psi}_{k, L}}{\left\|\boldsymbol{\psi}_{k, L}\right\|^{2}}
$$

Note that in this case, the norm of the estimated parameter vector is not constant in time anymore. In order to prevent the risk of numerical instabilities, we have to add an extra normalization step after (14). The main drawback of applying this relaxation step as a post process is that, not only the effect of noise, but also the speed of convergence are largely dependent on the choice of $\mu$. If this relaxation parameter is near to zero, the effect of noise is significantly reduced, but the convergence speed is slowed down. In counterpart, if $\mu$ is near to 1 , we observe the opposite effect. Thus, a good compromise must be made between convergence speed and measurement noise reduction. It is interesting to note that if $\mu=1$, the update relation (10) is recovered, and on the other hand, if $\mu=0$, we get $\hat{\tilde{\mathbf{h}}}_{k+1}=\hat{\tilde{\mathbf{h}}}_{1}$ for all $k$. This notion of compromise is graphically illustrated in Fig. 4 in the case where $\sigma_{b}^{2}=10^{-1}$.

In this example, we have succeeded in decreasing the average error level from about $10^{-1}$ to about $10^{-3}$ with a relaxation coefficient of 0.02 but the number of iterations has been raised by a factor of 100 .

\section{Conclusion}

In this contribution, we extended the LIMBO method to a more general context involving, besides the impulse response and the offset, the estimation of the gain of the system. In

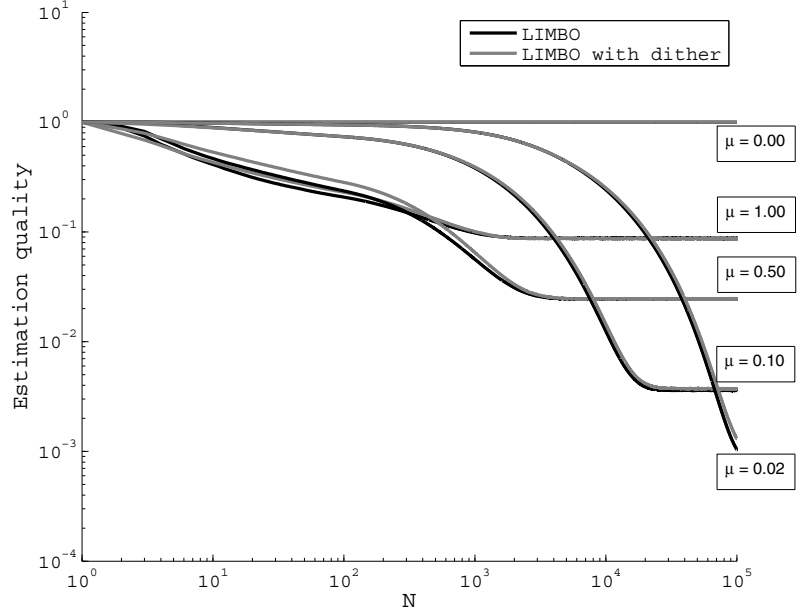

Fig. 4. Empirical mean of the quantities $\left(1-v_{k}\right)$ and $\left(1-\tilde{v}_{k}\right)$ for several values of $\mu$ considering a Gaussian distribution of the noise with $\sigma_{b}^{2}=10^{-1}$ and using a binary (Bernoulli) distributed input signal.

this manner, the true parameter vector was estimated instead of its normalized form. We thus overcame, by introducing a known dithering signal at the comparator input, the main drawback of LIMBO. Indeed, under its standard formulation, LIMBO was only able to estimate the parameter vector up to a positive multiplicative constant. We showed by Monte Carlo simulations that the performances of this new version in terms of convergence speed and estimation quality are similar to those obtained by LIMBO. In addition, a relaxation procedure was exposed in order to reduce the effect of measurement noise. Moreover, the implementation cost of this novel method remains very low, making it appropriate for use in the context of micro-electronics, as it was already the case for LIMBO. Finally, the convergence analysis of this method could be straightforwardly studied by following the same methodology as [14].

\section{REFERENCES}

[1] E. Colinet and J. Juillard, "A weighted least-squares approach to parameter estimation problems based on binary measurements," IEEE Transactions on Automatic Control, vol. 55, no. 1, pp. 148-152, 2010.

[2] R. V. de Plassche, CMOS Integrated Analog-to-Digital and Digital-toAnalog Converters. Springer-Verlag, 2005.

[3] T. Wigren, "Ode analysis and redesign in blind adaptation," IEEE Transactions on Automatic Control, vol. 42, no. 12, pp. 1742-1747, 1997.

[4] _ "Adaptive filtering using quantized output measurements," IEEE Transactions on Automatic Control, vol. 46, no. 12, pp. 3423-3426, 1998.

[5] L. Wang, J. Zhang, and G. Yin, "System identification using binary sensors," IEEE Transactions on Automatic Control, vol. 48, no. 11, pp. 1892-1907, 2003.

[6] L. Wang, G. Yin, and J. Zhang, "Joint identification of plant rational models and noise distribution functions using binary-valued observations," Automatica, vol. 42, no. 4, pp. 535-547, 2006.

[7] M. Negreiros, L. Carro, and A. Susin, "Ultimate low cost analog bist," in Proceedings of the 40th Design Automation Conference, New York, New York (USA), 2003, pp. 570-573.

[8] E. Rafajlowicz, "Linear systems identification from random threshold binary data," IEEE Transactions on Automatic Control, vol. 44, no. 8, pp. 2064-2070, 1996. 
[9] E.-W. Bai and J. R. Jr., "Towards identification of wiener systems with the least amount of a priori information on the nonlinearity," Automatica, vol. 44, no. 4, pp. 910-919, 2008

[10] F. Gustafsson and R. Karlsson, "Statistical results for system identification based on quantized observations," Automatica, vol. 45, no. 12, pp. 2794-2801, 2009.

[11] K. Jafari, J. Juillard, and E. Colinet, "A recursive system identification method based on binary measurements," in Proceedings of the 49th IEEE Conference on Decision and Control, Atlanta, Georgia (USA), 2010, pp. 1154-1158.

[12] J. Juillard, K. Jafari, and E. Colinet, "Asymptotic consistency of weighted least-square estimators for parameter estimation problems based on binary measurements," in Proceedings of the 15th IFAC Symposium on System Identification, Saint-Malo, France, 2009, pp. 72 77.

[13] O. Legendre, H. Bertin, O. Garel, H. Mathias, S. Megherbi, K. Jafari, J. Juillard, and E. Colinet, "A low-cost, built-in self-test method for resistive mems sensors," in Proceedings of Eurosensors XXV (Eurosensors'11), Athens, Greece, 2011, pp. 182-185.

[14] L. Bourgois and J. Juillard, "Convergence analysis of an online approach to parameter estimation problems based on binary noisy observations," in Proceedings of the 51st IEEE Conference on Decision and Control, Maui, Hawaii (USA), 2012, pp. 1506-1511. 